\title{
IMPACT OF IMMUNE DYSFUNCTION AND STRESS RELATED DISORDERS IN ENDOMETRIOSIS - A CASE-CONTROL STUDY
}

\section{Simone Subtil; Inês Ramalho; Sara Campos; Maria João Carvalho; Margarida Figueiredo-Dias \\ Gynecology Department, Coimbra University Hospital Center (CHUC), Portugal.}

Faculty of Medicine of Coimbra University, Portugal

\section{Problem statement}

Endometriosis is usually linked to significant emotional distress. Nevertheless the pathophysiology of this gynecological condition is not yet established and the contribution of higher stress levels is not clear enough. Moreover women with endometriosis are at increased risk for second disease as chronic fatigue syndrome, multiple sclerosis, lupus, rheumatoid arthritis and other autoimmune inflammatory related disorders. Allergies, asthma and eczema has been also reported as more prevalent among endometriosis patients.

\section{Methods}

Surgical diagnosed endometriosis patients $(\boldsymbol{n}=\mathbf{4 1 8})$

(Gynecology Department, Coimbra University Hospital Center :2006-2017).

Control group of 300 stratified healthy women.
Body mass index

Educational level

Auto-immune diseases

Allergies (food and drugs)

Stress-related disorders

(depression, anxiety or fibromyalgia)

\section{Results}

Age at diagnosis:

$37,2 \pm 8,1$ years [19-80]
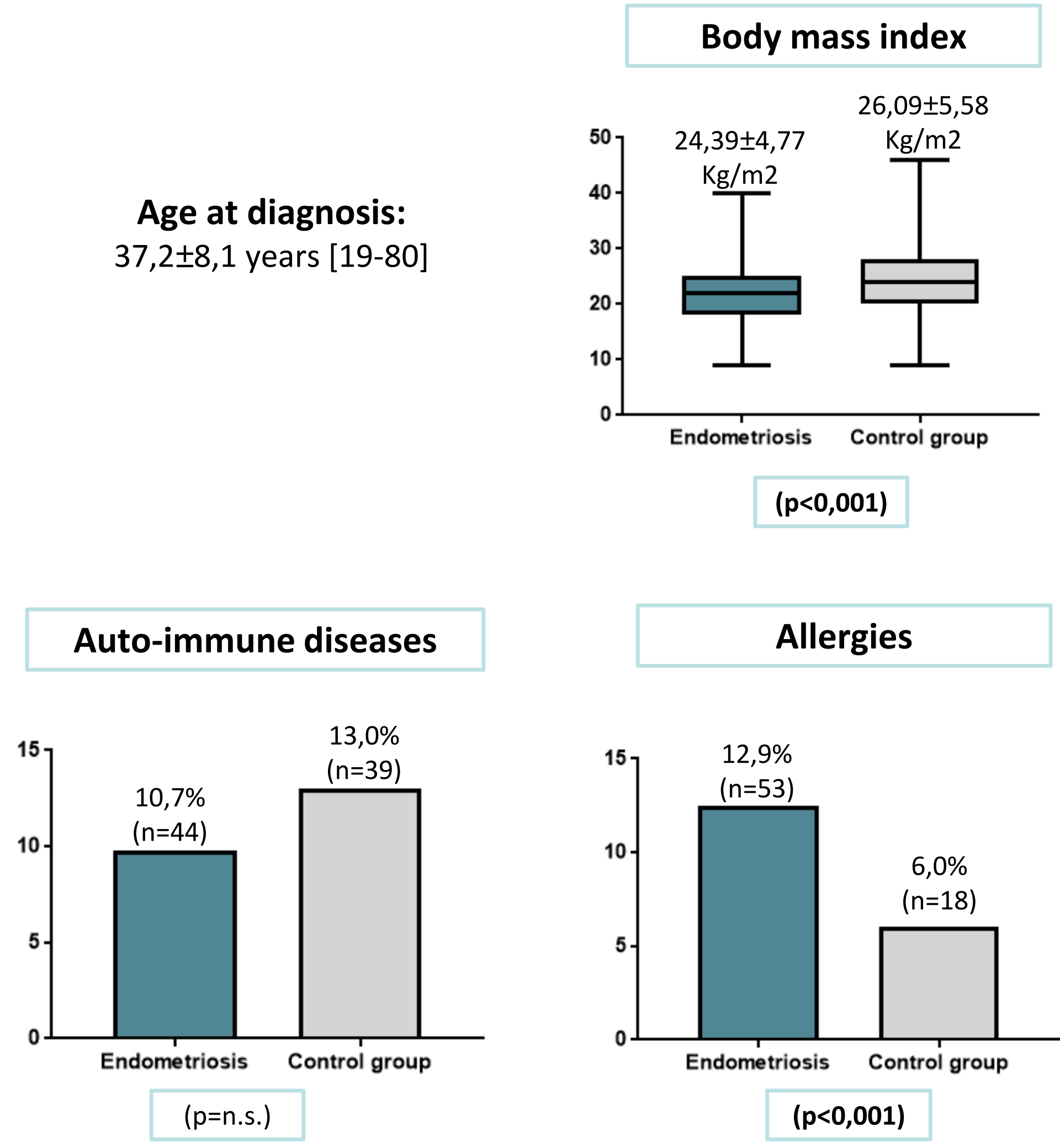

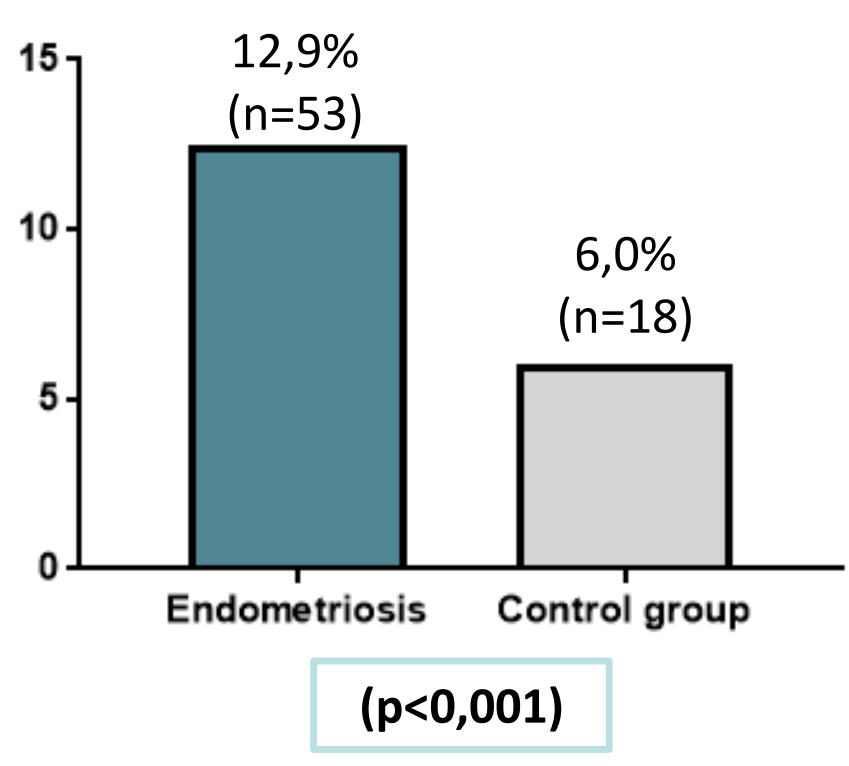

$(p<0,001)$

\section{Higher educational levels}



\section{Stress-related disorders}

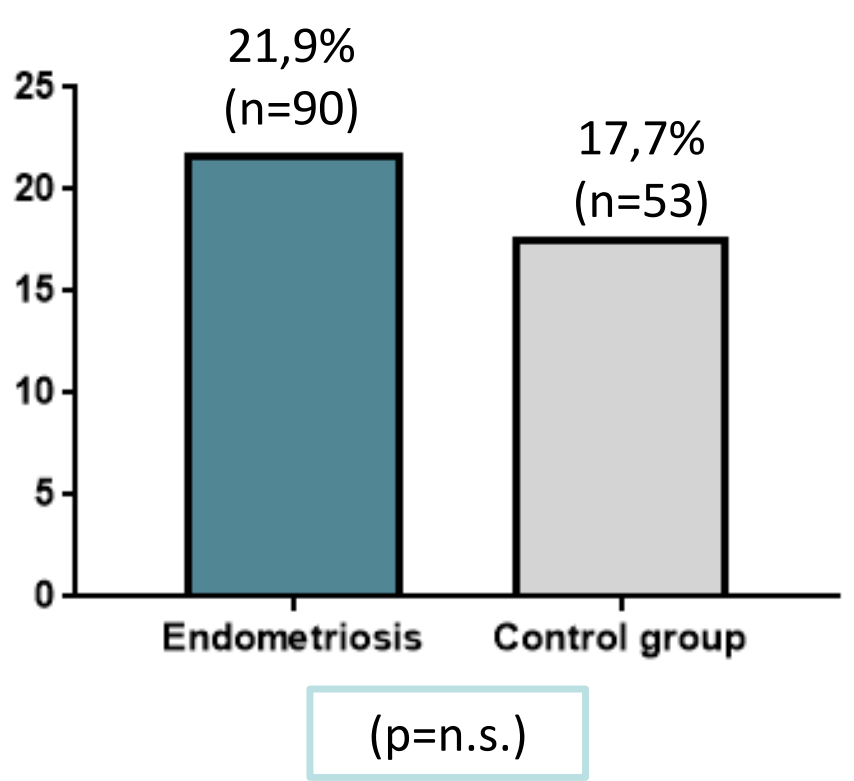

\section{Conclusions}

A possible link between endometriosis and higher educational level and severe allergies was established. Stress can lead to an altered immune response, promoting a favorable inflammatory environment for endometriotic implants survival and growth. However, we found no significant difference on what concerns autoimmune diseases or stress-related disorders. Further studies should be specifically designed in order to confirm these preliminary results. 